\title{
An Innovative Rotordynamical Model for Coupled Flexural-Torsional Vibrations in Rotating Machines
}

\author{
Filippo Cangioli, Alice Innocenti, Lorenzo Marini, \\ Enrico Meli, Luca Pugi, and Andrea Rindi \\ Department of Industrial Engineering \\ University of Florence \\ via di S.Marta 3, 50139 Florence, Italy \\ \{f.cangioli, alice.innocenti, lorenzo.marini, enrico.meli, luca.pugi, andrea. \\ rindi\}@unifi.it \\ http://http://www.dief.unifi.it/
}

\begin{abstract}
In the case of rotating machinery, for a long time most of studies has focused on a single form of vibration, hence flexural and torsional vibrations were usually studied in a separate way. Current design trends for rotating equipment (higher power transmission and efficiency by reducing weight and increasing operating speeds) set the assumption on treating flexural and torsional vibrations by separate and decoupled analysis much less accurate. This coupling usually exists, as it is caused by the most common malfunction conditions. Moreover, complex distributed inertial elements connected to the shaft may determine the coupling of flexural and lateral vibrations as well. In the paper, the authors present an accurate, innovative and fully coupled model for the evaluation of the dynamic behaviour of multi-rotor systems that has been conceived with the goal of investigating the flexural-torsional inter-action in rotor vibrations. The model, based on a finite element (FE) rotordynamics formulation with 6 DOFS for each node, is able to deal with long rotors characterised by complex topology, such as rotor with distributed inertias or connected simultaneously in several points. The rotor test case studied in the present research activity is a flywheel masses test bench for railway brakes. The effectiveness of the model in predicting the critical behaviour of the considered rotor system has been tested by means of experimental vibration data resulting from dedicated vibration tests campaign, performed in collaboration with Politecnico di Milano to assess the dynamical of the behavior of the machine.
\end{abstract}

Keywords: FE modelling, coupled flexural-torsional vibrations

\section{INTRODUCTION}

Understanding and accurate modelling of the complicated kinetic and dynamic phenomena characterizing rotating machinery represents a critical issue in the 
rotordynamics field. In the case of rotating machinery, for a long time most of studies has focused on a single form of vibration, hence lateral and torsional vibrations were usually studied in a separate way $[1-3]$ until some real applications [4] exhibited unpredicted critical resonances on the lateral vibrations characterized by the torsional mode frequency, demonstrating that decoupled flexural and torsional studies could not correctly predict some vibrations.

Current design trends for rotating equipment aim to get higher power transmission and higher efficiency by reducing weight and increasing operating speeds, setting the assumption on treating lateral and torsional vibrations by separate and decoupled analysis much less accurate. In fact, this coupling usually exists, as it is caused by the most common malfunction conditions, such as rotor unbalance, shaft misalignment or rotor-to-stator rubbing [5-7]. Coupling mechanisms in lateral-torsional dynamics may lead to rotor unstable behaviors, which mainly arise for rotor speed value where maps of lateral and torsional natural frequencies intersect each other. Hence, in those types of rotating machinery where the angular speed is subjected to high rate of changes, a correct identification of unstable regimes by means of coupled models is required [8]. Several research activities may be found in literature focusing on the coupling between lateral and torsional vibration dealing with both the development of new formulation (modelling aspect) and the investigation of the mechanisms inducing the vibration coupling. In [9] a 5 DOFS for each node finite element model for coupled lateral-torsional vibration of shaft system is established. In [5] a coupled lateral-torsional mathematical model of a Jeffcott's rotor is developed basing on the separation of the rotational rigid motion from its torsional deflection component. A fully coupled lumped model including lateral-torsional and the effect of the axial forces is developed in [10]. In [11] a finite element formulation of a rotor-bearing system is derived taking into account gyroscopic effects and lateral-torsional inertial interaction. A non-linear lumped model considering the six DOFS of the rotordynamics system is derived in [12] and the influence of both external and internal factors on the lateral-torsional coupling is investigated. Several sources causing excitation of lateral-torsional vibration are analysed in [4]. More specifically mathematical models are derived for the description of the lateral-torsional dynamic coupling due to unbalance, variable torques and rotor anisotropy. Another important source of flexural-lateral coupling in rotors is the presence of a gearbox, where lateral and torsional vibrations get coupled due to the tooth force. Many research activities are available in literature dealing with this topic $[13,6]$. Also misalignment due to flexible-coupling is an important source of coupled flexural-torsional behaviour. The coupling between lateral and torsional vibrations in rotors may also arise due to rotor cracks $[14,15]$, to the dynamics of blades in bladed rotors [7] or due to rotor-to-stator rubbing [15]. As far as the authors know, in the literature, even if several models concerning classical rotordynamics approaches may be found $[1,2,4,3]$, there is a substantial lack concerning models suitable for the coupled flexural-torsional analysis of multi-rotor systems characterised by complex topology, such as rotors with distributed elements. In the present paper, the authors introduce a fully coupled 
model developed with the aim of studying the lateral-torsional interaction in vibrations of complex rotordynamics systems; for this kind of systems coupling phenomena are difficult to be assessed and studied with classical rotordynamics elements. The model studies the dynamic behavior by means of a FE formulation characterized by 6 DOFS for each node. Based on the finite element formulation a dedicated tool has been developed in the MatLab with Comsol 4.4a environment. The rotor test case studied and modeled in the present work is a flywheel masses test bench for railway brakes. It has been adopted as case study for its complex configuration since its shaft-line consists effectively in a multi-rotor formed of multiple shafts group presenting several distributed inertial elements. Moreover, the particular operating conditions (transient characterized by high torsional and flexural applied moments) of the test bench may induce harmful stress state in the rotor structure and may also determines complex modes and coupled flexural-torsional vibrations.

The rotordynamics model developed by the authors has been validated by means of experimental data coming from a test campaign aimed at the evaluation of the vibration behaviour of the machinery. The test campaign has been performed by Politecnico di Milano, thanks to the instrumentation of the test bench by means of accelerometers to measure mechanical vibration signals in terms of acceleration characterizing the non-rotating parts of the machinery [16].

\section{GENERAL ARCHITECTURE OF THE MODEL}

The model proposed by the authors represents a systematic and practical approach to the rotor dynamics modelling issue and its main contribution to the research topic in rotors modelling, is due to the very general topology of the model. The present model is an accurate finite element model (FEM), characterized by 6 DOFS for each node. Furthermore, thanks to its numerical efficiency, the proposed model represents a good compromise between accuracy and computational effort, thus it may be used to perform the common dynamical investigation used in rotordynamics design phase. Moreover, traditional uncoupled, such as flexural (4 DOFS) or torsional (1 DOF) studies, or coupled such as axial-flexural-torsional (6 DOFS) or flexural-torsional (6 DOFS) analysis may be performed (see Fig. 1 ). The general architecture of the innovative model, which is represented in Fig. 1a), introduced by the authors is composed of three succeeding parts: the Physical Inputs module that concerns pre-processing phase, the Rotordynamics Model, representing the core of the proposed model, the Rotordynamics Analysis module, conceived for the post-processing of the results.

The Physical Inputs part(implemented in Matlab) is the module that contains all the physical inputs that must be provided to the model to perform the rotor discretisation and the dynamical analysis. The core of the innovative model introduced in the presented paper by the authors is the second part of the general architecture, the Rotordynamics Model, which is the module responsible for the model set-up and for the dynamical analysis. The Rotordynamics Model is a model, which is able to reproduce the dynamics of a generic multi- 


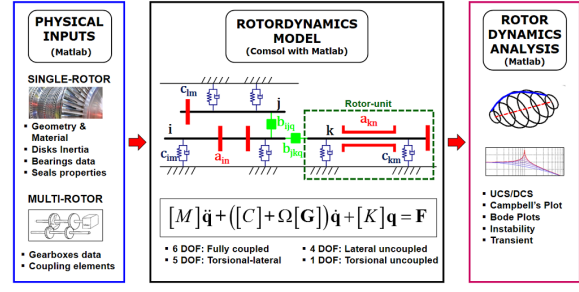

(a)

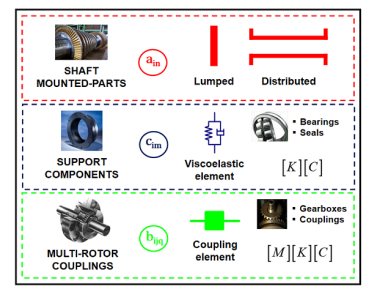

(b)

Fig. 1. General architecture of the model (a): scheme (b): Rotordynamics elements.

shaft rotor assembly, taking into account all the components that influence the response of the investigated rotor system Fig. 1b. The elementary unit of the Rotordynamics Model is represented by the Rotor Unit that is an accurate FEM model of a single-rotor formed of basic building blocks (rotordynamics elements). More specifically, it is the module responsible for describing the rotor mass-elastic properties by means of both classical and innovative rotor FEM elements such as shafts, disks, bearings and seals [1]. In Fig. 1b Rotor Units are conventionally denoted by means of indexes $i, j$ and $k$. The building blocks that must be joined together to form a complete Rotor unit may be distinguished according to the physical characteristics of a generic rotating assembly: 1. Shaft elements that give contribute both on stiffness and inertial property. 2. Mounted elements, representing disks or other massive components shrunk onto the shaft. In Fig. 1b mounted elements are conventionally indicated with $a_{i n}$, where subscripts $i$ and $n$ denote respectively the rotor and the disk index. Due to the high complexity characterising modern rotating systems, the necessity of generating detailed inertia distribution is increased. Therefore the Rotor unit proposed by the authors may represent two types of mounted elements: lumped mass or inertial distributed elements with complex topology. Lumped elements contribute only to inertial properties thus representing the disk elements of classical rotordynamics models. The inertial distributed elements are employed to model particular mounted elements and they may be used to easily reproduce different types of connection, which can be adopted to take into account the elastic effects of massive components. In particular distributed elements may be adopted to correctly model elements characterised by more complex shaft-torotor multiple-points connections. 3. Elasto-damping lumped elements $\left(c_{i m}\right.$ where $m$ is the connection index) are commonly employed to take into account the contribution that seals or bearings can give to the rotor vibration behaviour. In order to obtain a multi-rotor configuration several Rotor units may be used and connected each other thanks to innovative elements that model the common devices used for the power transmission. The linking elements, conventionally denoted by $b_{i j q}$ (where the $i$ and $j$ subscripts define the connected rotors, while the $q$ subscript is the multi-rotor connection index), that can be easily investigated thanks to the present model are the mechanical systems used to drive rotating 
machinery such as flexible couplings and gear-boxes. The developed model aims at overcoming modelling limitations relative to rotor with distributed inertias, such as rotor with distributed inertias or connected simultaneously in several points. The modelling of this kind of rotors may affect the dynamical behaviour of the system with particular concern to the flexural-torsional coupled vibrations. The model is able to study the influence of distributed rotors on the dynamics of an investigated system, thanks to a $\mathrm{FE}$ formulation with $3 \mathrm{D}$ beam that may shift among formulations characterised by 1, 4, 5 and 6 DOFS for each node. The Rotordynamics Model has been specifically developed to perform classical rotordynamics analysis, which can be very useful in designing, operating, and troubleshooting phases of rotating equipments. The entire Rotordynamics Model has been implemented in the Matlab with Comsol v.4.4a environment [17]. The third part is the the Rotordynamics analysis, responsible for the post-processing (Matlab environment).

\section{TEST CASE DESCRIPTION}

The test case studied in the current work is a dynamometric flywheel test bench, designed for the acceptance tests of railway brake components. It presents several distributed inertial elements characterised by a multi-point connection inducing complex dynamical behaviours (such as flexural-torsional vibrations). Fig. 2a shows a scheme of the studied rotor. The whole train is formed of six parts linked by means of flexible couplings: the electrical motor group driving the train, the four flywheel-shaft groups and the shaft supporting the disc brake to be tested. The electrical motor driving the shaft line is a $500 \mathrm{~kW}$ asynchronous

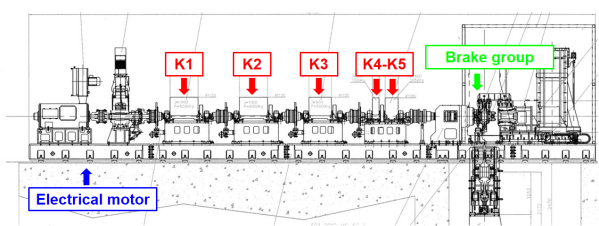

(a)

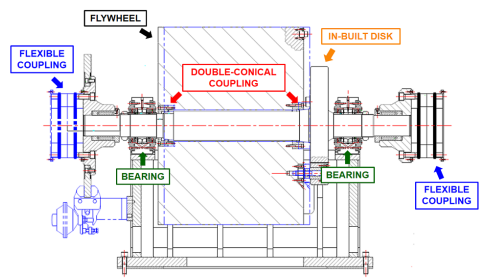

(b)

Fig. 2. dynamometric flywheel test bench (a): view (b): Layout of the K1 flywheel-shaft group.

three-phases motor, which has been designed for this particular application. The maximum rotating speed of the motor is $3000 \mathrm{rpm}$. The part of the studied test case responsible for the simulation of the mechanical inertia is based on a modular architecture, formed of four flywheel-groups, named K1, K2, K3 and K4 (see Fig. 2). The four modules have a similar geometrical layout and differ each other 
for the inertial properties. The total number $N_{\text {tot }}$ of flywheel is five, thus allowing $2^{N_{t o t}}=32$ possible combinations of the simulated inertia. The layout of a flywheel-shaft group,illustrated in Fig. 2b, is composed of: a steel forged flywheel, a steel transmission shaft with an in-built disk to engage the corresponding flywheel, an emergency disk-brake, two self-aligning roller bearings and a baseplate supporting the flywheel when it is disengaged. When the flywheel is translated to the engagement position (represented with the blue line in Fig. 2b), it is axially coupled with its supporting shaft by means of a double-conical coupling. The rotational locking to the shaft is then assured by fastening the flywheel to the inbuilt disk by means of three screws. The flywheel represents a distributed inertial elements with several shaft-to-rotor point connections, thus defining a complex topology rotor system. This kind of elements are difficult to be described and their mathematical modelling requires the complete definition of stiffness $[K]$ and damping $[C]$ properties of the geometrical coupling.

The two ends of each flywheel-shaft module are supported on two identical self-aligning spherical roller bearings. Dynamical properties of the self-aligning spherical roller bearings adopted in the present test case, have been provided by the bearing manufacturer according to the static load acting on them in the different configurations of the machine. In the investigated test case the shaft line is connected through 5 torsionally-stiff flexible couplings. The brake supporting group (BG) is the part of the test case on which the brake system to be tested (brake disc or train wheel) is mounted. The shaft of the brake group is thus subjected to high flexural and torsional loads and this aspect is particularly critical if combined with the presence of complex rotor elements. The brake supporting group is connected to the flywheels shaft line through a safety hydraulic coupling and of a torsiometer for the measurement of the braking torque.

\subsection{Description of the experimental data}

Several measurement campaigns have been executed on the studied test bench in collaboration with Politecnico of Milano. The simulation campaigns can be classified and conventionally named: Test campaign 1, Test campaign 2 and Test campaign 3. The measurement have been performed according to the guidelines provided by the ISO/10816 series Mechanical vibration Evaluation of machine vibration by measurements on non-rotating parts [16]. The test bench has been instrumented by means of: a set of vertical and lateral piezo-electric accelerometers, vertical and lateral servo-accelerometers and an encoder.

The dynamic behaviour of the studied test case has been investigated in different configurations of the machine and for several operating conditions. Moreover, the vibration of the test bench have been measured according to different working conditions: free and braking conditions and the tests executed during the measurement test campaigns are impulsive excitation tests, run tests that can be distinguished in free and braking runs. In the present paper experimental data coming from Test campaign 3 are investigated. Braking and free run tests 
have been performed during this test campaign, for sake of brevity, only the executed free tests are listed in Tab. 1.

Table 1. List of free tests performed in Test Campaign 3.

\begin{tabular}{|c|c|c|c|c|}
\hline & $\begin{array}{l}\text { Mounted } \\
\text { flywheels }\end{array}$ & $\begin{array}{l}\text { Test } \\
\text { type }\end{array}$ & $\begin{array}{l}\text { Total Inertia } \\
\quad\left(\mathrm{kg} \mathrm{m}^{2}\right)\end{array}$ & $\begin{array}{l}\text { Maximum speed } \\
(\mathrm{rpm})\end{array}$ \\
\hline Run 1 & - & Run Test & 200 & 3000 \\
\hline Run 2 & K5 & Run Test & 600 & 3000 \\
\hline Run 3 & K4 & Run Test & 400 & 3000 \\
\hline Run 4 & $\mathrm{~K} 4+\mathrm{K} 5$ & Run Test & 800 & 2800 \\
\hline Run 5 & K3 & Run Test & 1000 & 3000 \\
\hline Run 6 & $\mathrm{~K} 3+\mathrm{K} 5$ & Run Test & 1400 & 2900 \\
\hline Run 7 & $\mathrm{~K} 3+\mathrm{K} 4$ & Run Test & 1200 & 2900 \\
\hline Run 8 & $\mathrm{~K} 3+\mathrm{K} 4+\mathrm{K} 5$ & Run Test & 1600 & 2800 \\
\hline Run 9 & $\mathrm{~K} 2$ & Run Test & 1200 & 2400 \\
\hline Run 10 & K1 & Run Test & 1200 & 2400 \\
\hline Run 11 & $\mathrm{~K} 1+\mathrm{K} 3$ & Run Test & 2000 & 2500 \\
\hline Run 12 & $\mathrm{~K} 1+\mathrm{K} 2$ & Run Test & 2200 & 2300 \\
\hline Run 13 & $\mathrm{~K} 1+\mathrm{K} 2+\mathrm{K} 3$ & Run Test & 3000 & 2300 \\
\hline Run 13 & $\mathrm{~K} 1+\mathrm{K} 2+\mathrm{K} 3+\mathrm{K} 5$ & Run Test & 3000 & 2300 \\
\hline Run 13 & $\mathrm{~K} 1+\mathrm{K} 2+\mathrm{K} 3+\mathrm{K} 4+\mathrm{K} 5$ & Run Test & 3000 & 2300 \\
\hline
\end{tabular}

\section{PRELIMINARY VALIDATION OF THE MODEL}

The dynamometrical test bench investigated as case-study in the present paper is characterised by the presence of four flywheel elements representing distributed inertial elements with several shaft-to-rotor point of connections. This feature, the length of the shaft line and the number of the installed flexible-couplings matched with the particular operating conditions set the test bench as a critical application from the standpoint of flexural-torsional coupled dynamics. During the test campaign performed to assess the vibration level of the machine, the presence of an asynchronous component dependent on the particular installed flywheel configuration has been observed. A 5 DOFS model of the studied test bench has been realized to study the possible correlation of the non harmonic components with the coupling of flexural and torsional dynamics due to the presence of complex topology components in the machine.

In the present paper the results relative to the $\mathrm{K} 2$ machine configuration in terms of accelerations of the vibrations are shown considering the matching with the experimental data of free run tests of Test Campaign 3. The discretised model of the K2 multi-rotor configuration is formed of six Rotor units connected each other by five flexible-couplings. A schematic representation of the rotor system is illustrated in Fig. 3 .

The K2 configuration is modelled through 141 3D shaft elements with 6 DOFS per node that mathematically describes the multi-rotor shaft line formed by the motor group (MG), the flywheel-shafts groups and the brake supporting group (BG). The section properties are attributed according to real geometries 


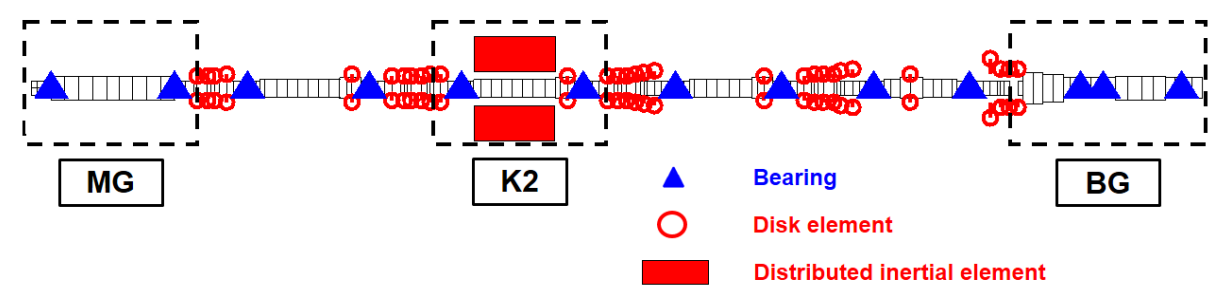

Fig. 3. Discretisation of the K2 configuration.

of the shafts. Concerning rigid disk elements, a total of 30 lumped mass have been added to nodes to take into account the inertial influence of the emergency brake disks, the in-built disks, the tested brake-disk and the flanges of the flexible-couplings. The lumped masses are placed in the nodes corresponding to their location on their shaft. In the developed model 13 bearings elements are introduced. The K2 flywheel is modelled as a distributed inertial element connected to the shaft in two points. The geometry coupling axially and torsionally the K2 flywheel to the shaft is described with different values of the $K_{K 2 S D I}^{c o n}(\Omega)$, $K_{K 2 S M O}^{c o n}(\Omega)$ and $C_{K 2 S M O}^{c o n}(\Omega), C_{K 2 S D I}^{c o n}(\Omega)$ matrices. To consider the influence of the centrifugal forces on the double-conical coupling the stiffness matrices is speed dependent. The torsionally-stiff flexible-couplings are introduced as general elasto-damping elements linking the single Rotor units. The inertial properties of the flexible-couplings are introduced by means of classical disk massive elements. In Fig. 4b the spectrograms of the vertical accelerations of the vibrations measured on the bearing housings of both K1 and K2 shaft groups during Free run test 9 in Tesc Campaign 3, is reported. The vertical axis represents time, the horizontal axis is frequency and the third dimension (represented by the intensity or color of each point in the image) denotes the amplitude of a defined frequency at a particular time. The four accelerometers exhibit clearly the $1 \mathrm{x}$ harmonic response in the three phase of the test: run-up, regime and run down. It must be noticed that the vertical acceleration measured on the K2 NDE bearing (the bearing that is nearest to the in-built disk for the torsional coupling of the flywheel to the shaft) shows also a response characterised by a costant frequency $\left(f_{K 2-N D E I}^{e x p}\right.$ around $\left.14 \mathrm{~Hz}\right)$ independent from the rotation regime of the machine. The Campbell Diagram of the investigated configuration is derived through a 5 DOFS formulation (axial vibration can be ignored if compared with the other vibration components) and considering a step of $100 \mathrm{rpm}$ is illustrated in Fig. 4a. The Campbell Diagram properly predicts three possible critical speeds $f_{K 2 m I}^{c o m p-s t}=13.14 f_{K 2 m I I}^{c o m p-s t}=20.21$ and $f_{K 2 ~}^{c o m I I I}=36.17$ in the operating range of the machine related to torsional natural frequencies. The experimental data (see Fig. 4bd) shows a dynamical amplification of the lateral response of the rotor characterised by a frequency value near to the one of the first critical speed $f_{K 2 m I}^{c o m p-r e s}=47.38 \mathrm{H}$ predicted by the Campbell's Diagram, hence demonstrating the capacity of the model in properly predicting coupled flexural-torsional frequencies of complex rotor models. 


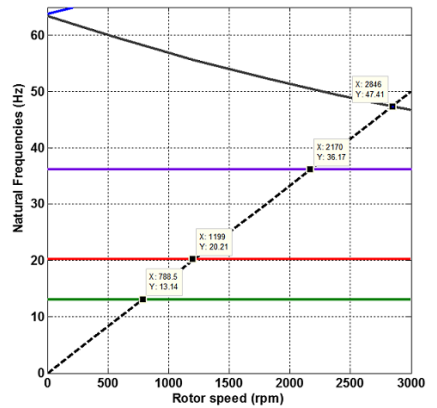

(a)
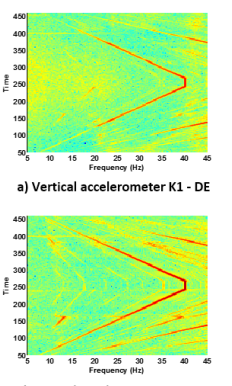

c) Vertical accelerometer K2 $-\mathrm{DE}$

(b)
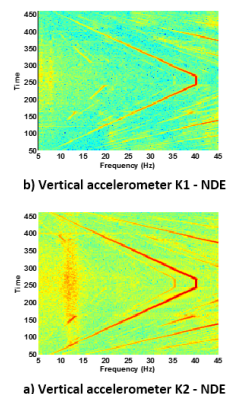

b)

Fig. 4. K2 configuration (a): Campbell's Diagram (b): Test Campaign 3 - Free Run test 9: vertical accelerometers on K1 and K2 flywheel-shaft groups.

\section{CONCLUSIONS}

In the paper, the authors introduce an accurate and general-purpose rotordynamics model for the analysis of multi-rotor systems, the aim of which consists in studying coupled flexural and torsional dynamics through complex modelling of rotors. It is based on a finite element rotor-dynamics formulation and it is able to perform standard uncoupled analysis and coupled study. The model is also able to manage long rotors characterised by complex topology, such as rotor with distributed inertias or connected to shaft in several points, elements that are particularly critical from a vibration coupling standpoint. A correct modelling of this kind of rotor is a fundamental aspect for the prediction of the critical behaviour of the investigated rotordynamics system, particularly when flexural-torsional dynamical couplings arise. The main innovative characteristics of the model consists in its general topology: it may reproduce rotors features by means of both classical and innovative rotor FE models. Multi-rotor linking elements (couplings and gearboxes) may be implemented and innovative elements may be also used for describing rotor non-standard components. The finite element formulation is derived considering 3D beams with 6 DOFS for each of the 2 node. The numerical efficiency of the presented model set it as a good compromise between accuracy and computational effort, thus it may be used to perform common rotor-dynamical investigation used in rotating machinery design. The case study analysed in the present research activity is a flywheel masses test bench for railway brakes. The rotordynamics model developed by the authors has been validated in a preliminary way by means of experimental data for the evaluation of the vibration levels of the machinery. Future developments of the present work will be based both on the modal analysis of other tested machine configurations of the present case study and on the comparison of the results coming from transient simulations with the vibration levels acquired during brake test. Simultaneously, other test cases, such as multi-rotor machines equipped with gearboxes, will be studied with the developed model and other 
causes of the flexural-torsional interaction will be analyzed. Authors would like to thank Prof. P. Pennacchi and Prof. A. Vania from Politecnico of Milano for their technical support during the research activity.

\section{References}

1. G. Ferraris, Rotordynamics prediction in engineering, no. v. 1 in Rotordynamics prediction in engineering, Wiley, 1990.

2. I. Friswell, Dynamics of Rotating Machines, Cambridge Aerospace Series, Cambridge University Press, 2010.

3. D. Childs, Turbomachinery rotordynamics: phenomena, modeling, and analysis, Wiley-Interscience publication, Wiley, 1993.

4. A. Muszynska, Rotordynamics, Dekker Mechanical Engineering, Taylor \& Francis, Dordrecht, Netherlands, 2010.

5. B. Al-Bedoor, Modeling the coupled torsional and lateral vibrations of unbalanced rotors, Computer Methods in Applied Mechanics and Engineering 190 (45) (2001) $5999-6008$.

6. M. Kita, T. Hataya, Y. Tokimasa, Study of a rotordynamic analysis method that considers torsional and lateral coupled vibrations in compressor trains with a gearbox, Proceedings of the thirty-sixth turbomachinery symposium 299 (12) (2007) $31-37$.

7. Y.-J. Chiu, D.-Z. Chen, The coupled vibration in a rotating multi-disk rotor system, International Journal of Mechanical Sciences 53 (1) (2011) 1 - 10.

8. Z. Gosiewski, Analysis of coupling mechanism in lateral/torsional rotor vibrations, Journal of theoretical and applied mechanics 46 (4) (2008) 829-844.

9. Q. H. Qin, C. X. Mao.

10. R. Sukkar, A. Yigit, Analysis of fully coupled torsional and lateral vibrations of unbalanced rotors subjected to axial loads, Kuwait J. Sci. Eng (35) (2008) 143 170.

11. M. Mohiuddin, Y. Khulief, Coupled bending torsional vibration of rotors using finite element, Journal of Sound and Vibration 223 (2) (1999) $297-316$.

12. Z. Yuan, F. Chu, Y. Lin, External and internal coupling effects of rotor's bending and torsional vibrations under unbalances, Journal of Sound and Vibration 299 (12) (2007) $339-347$.

13. J. Rao, T. Shiau, J. Chang, Theoretical analysis of lateral response due to torsional excitation of geared rotors, Mechanism and Machine Theory 33 (6) (1998) 761 783.

14. N. Bachschmid, P. Pennacchi, E. Tanzi, Cracked Rotors: A Survey on Static and Dynamic Behaviour Including Modelling and Diagnosis, Springer-Verlag, 2010.

15. T. H. Patel, A. K. Darpe, Coupled bending-torsional vibration analysis of rotor with rub and crack, Journal of Sound and Vibration 326 (35) (2009) $740-752$.

16. I. 10816-1, Mechanical vibration, evaluation of machine vibration by measurements on non-rotating parts - part 1: General guidelines (December 1995).

17. COMSOL Multiphysics ${ }^{\circledR}$ : Reference Manual, www.comsol.com (1998-2013). 